\title{
Adipose-derived mesenchymal stem cells (ADMSCs) protect against hyperglycemia and hyperlipidemia-induced heart failure by inhibiting autophagy-related apoptosis
}

$\mathrm{Xu} \mathbf{X u}$

Shanghai Tenth People's Hospital

Lingyun Tao

Shanghai laboratory animal research center

Jie zhou

shanghai laboratory animal research center

Jing Ni ( $\sim 15900750278 @ 163 . c o m$ )

Tongji University School of Medicine

Research

Keywords: heart failure, hyperglycemia, hyperlipidemia, ADMSCs, autophagy

Posted Date: October 1st, 2021

DOl: https://doi.org/10.21203/rs.3.rs-864688/v1

License: (c) (i) This work is licensed under a Creative Commons Attribution 4.0 International License.

Read Full License 


\section{Abstract \\ Background}

Mesenchymal stem cells (MSCs), a type of seed cell, are expected to improve impaired diabetic cardiac function. Inflammation and autophagy play an important role in the development of metabolic disorderinduced heart failure. The aim of this work was to assess the effect of adipose-derived mesenchymal stem cells (ADMSCs) on metabolic disorder-induced heart failure and the underlying mechanisms.

\section{Methods}

Eight weeks old male C57BL/6 mice were randomly divided into three groups: normal chow mice (NC group), high-fat diet-fed and streptozotocin-treated mice (HFD+STZ group) and mice administered with ADMSCs-once per week for 3 months after the mice were treated with HFD+STZ (ADMSCs+HFD+STZ group). Once a week lipid and glucose levels were measured and echocardiography was performed. Immunohistochemistry was used to detect the adhesion of macrophages in heart tissue in all three groups. Inflammatory cytokines such as interleukin-1 $\beta$ (IL-1 $\beta$ ), tumor necrosis factor a (TNFa), interleukin6 (IL-6) and interleukin-8 (CXCL-15) were measured by western blot or RT-qPCR. In vitro, H9c2 cardiomyocytes were stimulated by $33 \mathrm{mM}$ glucose in the presence or absence of IL-1 $\beta$. Transmission electron microscopy, mRFG-GFP-LC3 assays and flow cytometry were used to investigate autophagyrelated apoptosis in $\mathrm{H} 9 \mathrm{c} 2$ cells.

\section{Results}

HFD+STZ treated mice presented significant cardiac hypertrophy, body weight loss, hyperglycemia and hyperlipidemia. However, these changes were remarkably reversed by the administration of ADMSCs. The administration of ADMSCs also remits histological alterations and deposition of collagen in the heart tissue. Furthermore, ADMSCs downregulated the adhesion of macrophages in heart tissue. More importantly, IL-1 $\beta$ from macrophages increased the autophagy of myocardial cells stimulated with high glucose, which eventually led to apoptosis and cardiac dysfunction.

\section{Conclusions}

This study confirmed that ADMSCs may have potential for use in improving cardiac function by restraining autophagy and apoptosis of myocardial cells. We also identified the roles of the IL-1 $\beta$ in hyperglycemia- and hyperlipidemia-induced cardiac injuries, which may be a key factor for diabetic complications.

\section{Introduction}


Previously, the Framingham study indicated that men and women with diabetes mellitus (DM) have a respective two and five-fold higher risk of developing heart failure (HF) than age matched controls [1]. Other clinical studies have also proved that patients with diabetes have LV diastolic dysfunction rather than LV systolic dysfunction in both T1DM and T2DM patients[2] and animal models[3]. These eventually led to the occurrence of asymptomatic heart disease in diabetic populations. However, a recent study from the Mayo Clinic with a larger patient population holds a different opinion. They have demonstrated a slightly lower left ventricular ejection fraction (LVEF) in diabetic patients compared to normal people. More importantly, patients with decreased LVEF may have poor survival even with diabetic asymptomatic heart disease[4]. Thus, it is important to investigate a possible strategy to improve the cardiac function of patients with DM.

It has been widely recognized that hyperglycemia and hyperlipidemia may be the main metabolic reasons for the clinical progression of cardiovascular diseases (CVD), including heart failure[5]. Hyperlipidemia is also considered as a major risk factor for CVD in type II diabetes mellitus[6, 7].An animal model also indicated that blood lipid lowering could inhibit pressure overload (transverse aortic constriction) [8] or myocardial infarction-induced heart failure[9] by promoting $\mathrm{Ca} 2+$ induced permeability transition in mitochondria. Here, we construct an animal model to investigate the role of hyperglycemia- and hyperlipidemia-induced HF with reduced left ventricular ejection fraction (HFrEF).

Mesenchymal stem cells (MSCs) are the most common cells used to treat cardiac diseases[10, 11]. Recent works have found that MSCs could enhance cardiac function and reduce the size of myocardial infarction by interfering with autophagy flow in an animal model[ $[12,13]$. There are different kinds of MSCs, including bone marrow-derived mesenchymal stem cells (BMSCs) and adipose-derived mesenchymal stem cells (ADMSCs). The content of ADMSCs is approximately 1:1500 and is more than BMSCs[14]. ADMSCs can be acquired conveniently and have multi-lineage capacity, which differentiate into adipose, bone, cartilage, cardiac muscle and epithelial cell types[15, 16]. ADMSCs are capable of inhibiting oxidative stress and inflammation by different mechanisms in experimental diabetic models[8, 17]. Previous studies have shown that ADMSCs decrease the expression of pro-inflammatory cytokines such as TNF- $a$ and IL-6 and ameliorate systematic inflammation in septic rats[18]. According to Sun $M$ et al., ADMSCs could decrease STAT3 and STAT6 expression and exert an anti-inflammatory role due to their effect on $M \varphi$ to $M 2 b / c-M \varphi[8]$. Thus, it is reasonable to hypothesize that ADMSCs could be used to protect the cardiac function of hyperglycemia- and hyperlipidemia-induced HFrEF as well.

\section{Methods}

\section{Isolation and Culture of ADMSCs}

SD rats were purchased from Shanghai Research Center of the Southern model organisms (China). ADSCs were isolated from the subcutaneous adipose tissue of 8 to 12-week-old SD rats. Briefly, adipose tissue was obtained from the subcutaneous fat pad around the ilium of the rats and was washed in phosphate-buffered saline (PBS) (CORNING, Manassas, USA) containing 1\% Penicillin-Streptomycin囚PS区 
solution (HyClone, Pasching, Austria). We minced the fat pad into pieces and then lysed in Dulbecco's modified Eagle's medium (DMEM) (Thermo Fisher, Suzhou, China) containing $1 \mathrm{mg} / \mathrm{ml}$ collagenase I (YEASEN, Shanghai, China) and $1 \% \mathrm{PS}$ at $37^{\circ} \mathrm{C}$ for $30 \mathrm{~min}$. The digested tissue was filtered through a sterile $100 \mu \mathrm{m}$ nylon mesh (Corning, NY, USA), and centrifuged at $1800 \mathrm{rpm}$ for $5 \mathrm{~min}$. After that, we discarded the supernatant and resuspended the pellet with culture medium twice. ADSCs were seeded onto culture dishes with growth medium DME/F-12 (HyClone, GE Healthcare Life Sciences, Logan, Utah, USA) containing $10 \%$ fetal bovine serum (FBS) (Sigma-Aldrich) and $1 \% \mathrm{PS}$ at $37^{\circ} \mathrm{C}$ in a humidified atmosphere of $5 \% \mathrm{CO}_{2}$ before being used for analysis.

\section{Animal Study}

8-week-old male C57BL/6 mice were purchased from Shanghai Research Center of the Southern model organisms (China). Type I diabetes was induced with a dose of streptozotocin (STZ, 70mg/kg in $0.1 \mathrm{~mol} / \mathrm{L}$ citrate buffer solution; Sigma-aldrich, St. Louis, Missouri, USA) intraperitoneally injected once every two days. Eight weeks later, the blood glucose levels (fasting blood glucose over $16.6 \mathrm{mmol} / \mathrm{L}$ were considered as diabetic) were measured. The diabetic mice were injected via the tail vein with ADMSCs $\left(2 \times 10^{6}\right)$ or saline for 12 weeks. Body weight, random blood glucose levels and blood lipid levels as well as echocardiography were tested at the time points. 30 weeks after the model establishment, all animals were sacrificed, and heart tissue was isolated for further analysis.

\section{Echocardiography}

Echocardiography was performed using a Vevo 2100 High Resolution Imaging System (Visual Sonics, Toronto, Canada). Briefly, mice were anesthetized by isoflurane $(1 \%)$ and placed on a $37^{\circ} \mathrm{C}$ platform. Parasternal long axis (PSLAX) images were acquired and analyzed with VevoStrain software. Using the M-mode images, the diameters of the left ventricle (LV) at the end of diastole (LVEDD) and systole (LVESD), LV fractional shortening (FS), and LV ejection fraction (EF) were calculated by the machine.

\section{Cell culture and stimulation}

H9c2 cardiomyocytes were purchased from the Institute of Biochemistry and Cell Biology (Shanghai, China). Cells were incubated in DMEM containing 10\% FBS and 1\% PS. For high glucose administration, cells were incubated with culture media containing $33 \mathrm{mM}$ glucose for 72 hours. H9c2 cardiomyocytes were treated with either high glucose (HG,33mM), IL-1 $(20 \mathrm{ng} / \mathrm{ml}$, popertech, cat\#400-01B) or a combination of IL-1 $\beta$ and HG after they were starved for 3 hours. Untreated cells were used as a negative control.

\section{Histopathology staining}


Myocardial tissue was fixed with 4\% paraformaldehyde and dehydrated and embedded in paraffin. Next, the samples were cut into 5 - $\mu \mathrm{m}$-thick sections. Heart sections were stained with hematoxylin-eosin (H\&E), Masson's trichrome and wheat germ agglutinin (WGA). The muscle cells with clear nuclei and clear sarcolemma were selected for analysis of muscle cell cross-sectional area (CSA) in H\&E and WGA staining. Fibrosis was detected on the heart tissue by Mason trichrome staining. Four microscopic photographs of every section were shot randomly and selected by NIS-elements v3.0 (Nikon, Japan). Cardiac CSA and myocardial fibrosis were quantified by Image-ProPlus6.0 software (Media Cybernetics, Silver Spring, Maryland, USA). Date is expressed as mean \pm standard deviation.

\section{Immunohistochemistry}

Myocardial tissues were fixed in $4 \%$ paraformaldehyde and then embedded in paraffin after dehydration. Next, the samples were cut into $5-\mu \mathrm{m}$-thick sections. Following this, the sections were deparaffinized and stained with F4/80 (diluted concentration 1:200, Servicebio, Wuhan, China). For IHC analysis, each section was captured by at least five images by NIS-elements v3.0 (Nikon, Japan) and all images were quantified using Image-ProPlus6.0 software (Media Cybernetics, Silver Spring, Maryland, USA).

Percentages of positive stains for F4/80 within the fields were averaged for each mouse and then for each group, as described before. Date is expressed as mean \pm standard deviation.

\section{Western blotting analysis}

Total protein was extracted from mouse hearts and the levels were determined using a BCA Protein Assay Kit (Thermo Scientific, Rockford, Illinois, USA). Equal amounts of total protein $(50 \mu \mathrm{g})$ were loaded and then separated by sodium dodecyl sulfate polyacrylamide gel electrophoresis (SDS-PAGE) and transferred to PVDF membranes (Thermo Scientific, Rockford, Illinois, USA). The membranes were then blocked in $5 \%$ BSA (YEASEN, Shanghai, China) for $1 \mathrm{~h}$, followed by overnight incubation at $4{ }^{\circ} \mathrm{C}$ with the following primary antibodies: IL-1 $\beta$ (cat: mAb \#12242, diluted concentration 1:1000, Cell Signaling Technology, Danvers, MA, USA), P62 (cat】ab109012, diluted concentration 1:800, abcam, England), LC3I/II (cat: ab62721, diluted concentration 1:500, abcam, England), and DAPDH (cat: ABS16, diluted concentration 1:10000, EMD Millipore Corp, USA). The secondary antibodies were horseradish peroxidase (HRP)-linked-antibody, anti-rabbit IgG (diluted concentration 1:5000, Cell Signaling Technology, Danvers, MA, USA) and HRP-labeled-antibody, Goat Anti-Mouse IgG (diluted concentration 1:5000, Biosharp Life Sciences, Hefei, China). Blots were processed for enhanced chemifluorescence using a Pierce ECL western blotting substrate (Tanon, Shanghai, China). Images were collected using ChemiDoc ${ }^{\text {TM }} \mathrm{MP}$ Imaging System (Bio-Rad, Hercules, CA, USA). Bands on immunoblots were quantified by densitometry using Image Lab software (version 6.0, Bio-Rad).

\section{Real-time quantitative polymerase chain reaction (RT-qPCR)}


Total RNA was purified from mouse hearts with TRIZol (Thermo Scientific, Rockford, Illinois, USA) following the manufacturer's protocol. The total RNA was used for reverse-transcription and amplified using a One Step SYBR RT-PCR Kit (TaKaRa, Shiga, Japan). Following this, the cDNA was amplified with SYBR Premix Ex Taq (KAPA, Cape, South Africa) by a Real-Time PCR System (Roche, Basel, Swiss). GAPDH was used as the internal control. The sequences of the primers for each gene detected are listed in Supplementary Table 1. The PCR program was as follows: $95^{\circ} \mathrm{C}, 10 \mathrm{~min} ; 95^{\circ} \mathrm{C}, 5 \mathrm{~s}, 60^{\circ} \mathrm{C}, 34 \mathrm{~s}$ for a total of 40 cycles. The expression levels of target genes were determined using the $2^{-\triangle \Delta C T}$ method.

\section{Annexin V-FITC/PI staining and flow cytometry}

H9c2 cells were treated as described above and were stained by using FITC Annexin V/Dead Cell Apoptosis Kit (cat: v13242, Invitrogen,USA) according to the manufacturer's instructions. Cells were then diluted in the dilution buffer and the apoptotic cells were measured by a BD FACS Calibur Flow Cytometer (BD Biosciences, Heidelberg, Germany) in a total of 10,000 counted cells.

\section{Transmission electron microscope and observation of autophagosome}

Briefly, the cell medium was removed and cells were washed with 0.1 cacodylate buffer and fixed with a solution containing $3 \%$ glutaraldehyde and $2 \%$ paraformaldehyde in PBS. Later, the rest of the procedure was conducted using the standard protocol. Transmission electron microscope (JEM-1400PLUS) was used to obtain the images.

\section{mRFG-GFP-LC3 assay}

H9c2 cardiomyocytes were seeded into a six-well-plate to reach about $60 \%$ confluence. Later, they were divided into 4 groups (NC group, HG group, IL-1 $\beta$ group, HG+ IL-1 $\beta$ group) and transfected with adenovirus (20 MOI, HBAD-mRFP-GFP-LC3, Hanbio, Shanghai, China) for 48 hours. In brief, cells were fixed in $4 \%$ paraformaldehyde for 10 mins and were washed with PBS three times. Cells were sealed by $10 \%$ glycerinum to limit fluorescence quenching. Leica DMI6000 was used to detect related fluorescence. GFP signals were observed in lysosomal acidic conditions, whereas mRFP fluorescence was relatively stable. Therefore, this tandem-tagged fluorescent protein showed yellow dots in the autophagosomes, while red was exhibited in lysosomes only. Quantification of GFP and mRFP fluorescent dots and the overlay between the two different signals were recorded and analyzed using ImageJ software.

\section{Statistical analysis}


All data are shown as the mean \pm SEM. Student's t-test (two-sided), one-way ANOVA or two-way ANOVA followed by Bonferroni's pos-hoc test were used to calculate $P$ value accordingly. Error bars represented standard errors, and numbers of experiments $(n)$ were as indicated. $\mathrm{P}<0.05$ was regarded as significant. All statistical analyses were performed using SPSS 20.0 software.

\section{Results}

Adipose -derived mesenchymal stem cells (ADMSCs) improved glycometabolism and lipid metabolism in high-fat diet/STZ-induced diabetic mice.

In order to investigate the potential of adipose-derived mesenchymal stem cells (ADMSCs) in mice with hyperglycemia and hyperlipidemia, three-month-old male C57BL/ 6 mice treated with STZ were fed either a high fat (HF) or normal chow (NC). Later, ADMSCs were injected into the STZ+HF group mice by tail vein injection as described in the methods section. After 8 weeks, STZ-induced diabetic mice fed with a HF diet exhibited slightly decreased body weight compared to the NC group mice. The body weight barely changed in the ADMSCs+HFD+STZ treated mice (Fig. 1A). Accordingly, the serum glucose level of $\mathrm{HF}+\mathrm{STZ}$ treated mice was higher than NC group mice.T Treatment with ADMSCs could improve the glycometabolism of HFD+STZ treated mice as shown in Fig. 1B. These mice had abnormally high serum glucose levels, but their serum glucose was reduced by the injection of ADMSCs. The triglyceride levels of ADMSCs+HFD+STZ treated mice also decreased. The enhanced serum cholesterol, high-density lipoprotein and low-density lipoprotein in the HFD+STZ group were unchanged after ADMSCs treatment (Fig. 1C-1F). In conclusion, injection of ADMSCs could greatly ameliorate hyperglycemia and hyperlipidemia in STZ-induced diabetic mice fed a HF diet.

\section{Adipose-derived mesenchymal stem cells (ADMSCs) alleviated heart failure of high-fat diet/STZ-induced diabetic mice.}

To explore whether ADMSCs could alleviate heart failure in HFD+STZ induced mice with metabolic disorder or not, these mice were subject echocardiograms to evaluate their cardiac function. Although the harvested hearts have no distinguished differences between groups, the thickness of the ventricular walls increased in HFD+STZ treated mice. The administration of ADMSCs restricted hypertrophic ventricular wall thickness in HFD+STZ treated mice (Fig. 2A). The data obtained from the echocardiograms verified a recovery of the cardiac function in ADMSCs injected HFD+STZ mice (Fig. 2B). Importantly, heart rates of mice were maintained at the same level with or without treatment (Fig. 2C). It could be observed that the HFD+STZ mice have declined cardiac function with decreased ejection function (EF, Fig. 2D), left ventricular short-axis shortening rate (FS, Fig. 2E) and an increased left ventricular internal dimension (LVID, Fig. 2F) compared to sham groups. The data from the HFD+STZ mice injected with ADMSCs showed that ADMSCs could promote EF, FS and decrease LVID compared with mice without administration of ADMSCs (Fig. 2D-2G). The HE staining of cardiac cross sections revealed that HFD+STZ mice have more vacuolization of cardiomyocytes. Importantly, the administration of ADMSCs eliminated these pathologic changes (Fig. 2H). Furthermore, the pictures taken from Massonstained 
cardiac cross sections proved that the deteriorated cardiac function could be attributed to the serious fibrosis of cardiac tissue in HFD+STZ mice. Meanwhile, the administration of ADMSCs in the HFD+STZ group mice greatly suppressed the fibration of heart tissue (Fig. 2J-2I). In line with these results, WAG staining demonstrated that ADMSCs may inhibit the enlargement of cardiomyocytes observed in HFD+STZ mice. Taken together, our results have clarified that ADMSCs could alleviate the cardiac dysfunction seen in HFD+STZ mice.

\section{Adipose-derived mesenchymal stem cells (ADMSCs) suppressed adhesion of macrophages and the secretion of IL-1 $\beta$ in vitro and in vivo.}

Recent studies have revealed that ADMSCs could suppress inflammation by regulating the polarization of macrophages and regulating the population of $M 1$ and $M 2$ macrophages[19, 20]. Also, macrophages have been indicated in cardiac remodeling in HF and were considered a clinical target of HF [21, 22]. IF staining was done to figure out the effect of ADMSCs on macrophages and the progression of HF. It could be observed that the F4/80 positive cells in the cardiac tissue of HFD+STZ-induced diabetic mice increased. The treatment of ADMSCs markedly inhibited the adhesion of macrophages in HFD+STZ mice (Fig. 3A-3B). Consistent with this result, the enhanced secretion of IL-1 $\beta$ was also decreased due to the limitation of macrophages in cardiac tissue with the help of ADMSCs (Fig. 3C-3D). The mRNA levels of other inflammatory cytokines such as tumor necrosis factor a (TNFa), interleukin-6 (IL-6) and interleukin-8 (CXCL15) were also decreased by the administration of ADMSCs (Fig. 3E-3G). Above all, it is reasonable to suspect that ADMSCs minimised the progression of heart failure by reducing inflammation and related cytokines such as IL-1 $\beta$ from macrophages.

\section{IL-1 $\beta$ enhanced diabetic cardiomyopathy by inhibiting autophagy and increasing apoptosis in $\mathrm{H} 9 \mathrm{c} 2$ cells.}

IL-1 $\beta$ is a cytokine believed to play a role in the acceleration of heart failure[23, 24]. Canakinumab, an IL$1 \beta$ inhibitor, has been tested in clinical trial to prevent hospitalization for heart failure[24]. However, the mechanism of IL-1 $\beta$ to improve the occurrence of heart failure was still unascertained. The results have indicated ADMSCs could decrease the distribution of IL-1 $\beta$ in the heart. HFD+STZ-induced diabetic mice exhibited severe fibration but a slight enlargement of myocardial hypertrophy in cardiac tissue. This phenomenon suggested the possibility of a reduced number of cardiomyocytes in the HFD+SZT mice. $\mathrm{H} 9 \mathrm{c} 2$ cells were stimulated with IL-1 $\beta$ and high glucose $(\mathrm{HG})$ at the same time for 72 hours. IL-1 1 + HG treated cells have increased apoptosis in all cells compared to IL-1 1 - or HG-stimulated cells (Fig. 4A-4B). The images obtained from transmission electron microscope showed more autophagic vacuolization in the IL-1 $\beta+$ HG stimulated group than other two groups (Fig. 4C). In addition, the protein expression of p62 and LC3I/II were measured by immunblot to monitor autophagic flux. IL-1 $\beta+\mathrm{HG}$ promoted the expression of LC3I/II and inhibited the expression of LC3 I/II and p62 which suggested that autophagy was enhanced in these cells. (Fig. 4D-4E). To confirm these results, tandem fluorescent mRFG-GFP-LC3 assay was performed to detect the autophagic flux. IL-1 $\beta+$ HG group cells have increased red foci which proved a weaker autophagic flux in the cells compared to other controls (Fig. 5A-5C). Thus, ADMSCs might prevent diabetic cardiomyopathy by promoting autophagy and apoptosis in IL-1 $\beta+$ HG stimulated cells. 


\section{Discussion}

Previously, it has been well known that ADMSCs can differentiate into adipose, bone, cartilage and epithelial cell types[12, 13]. Our data have proved that the injection of ADMSCs into HFD+STZ mice greatly prevented the progression of HF compared to sham group mice. However, ADMSCs might not be distributed into the heart directly which means that they might insert their function with the help of other cell types[25,26]. This was confirmed by the higher positive F4/80 staining of macrophages in the ADMSCs-treated group. Macrophages have been indicated in HF, particularly in myocardial infarction (MI)-induced HF[21]. They were the main contributors to the inflammatory and fibrotic processes in HF. Moreover, DM and insulin resistance were also associated with progression of HF[27, 28]. Our data provided a clue that ADMSCs have the potential to attract macrophages into the heart tissue of hyperglycemia- and hyperlipidemia-induced HF. Also, macrophages have different subtypes, such as M1, M2, tumor-associated macrophages (TAM), CD169』macrophages and TCR』macrophages[29, 30]. Our results have verified that the increased macrophages seen in heart tissue of the HFD+STZ mice were M1type macrophages. These macrophages may release inflammatory cytokines, such as IL-1 $\beta$ and TNFa. Nevertheless, precise classification should be performed by flow cytometric sorting as M1-type macrophages express CD14, CD11b, CD68 and MAC-1/MAC-3.

Also, it should be noted that the distribution of ADMSCs was not clear as they were not labeled to trace their confluence in particular organs. Earlier studies have demonstrated the distribution of ADMSCs was based on the type of injury[31, 32]. The majority of ADMSCs were attracted to damaged tissue and related regions with lack of blood and nutrition. Therefore, further experiments should be done to confirm how they promote the adhesion of macrophages in the heart.

Furthermore, the enrichment of macrophages in the heart tissue of HFD+STZ mice also increased IL-1 $\beta$. Myocardial cells exposed to the microenvironment of hyperglycemia and hyperlipidemia lacked proper of supply of nutrients. They relied on the autophagy pathway to save energy and keep themselves from apoptosis[1,33]. Lee et al. have verified ATG7 (autophagy-related gene) knockout mice could not survive over 12 hours because they showed their lack of nutrition supply[34]. Yet, there is controversy about the role of inflammation on autophagy. Some researchers insist that autophagy could accelerate inflammation[35, 36]. Other researchers hold the opposite views that hyperglycemia and hyperlipidemia could decrease the inflammation and apoptosis of myocardial cells by promoting autophagy[37, 38]. In our study, we noticed that inflammatory cytokines such as IL-1 $\beta$ could activate autophagy of H9c2 cells and increase the degree of autophagy in co-existence with hyperglycemia and the improvement eventually aggregated the apoptosis of $\mathrm{H} 9 \mathrm{c} 2$ cells. In conclusion, our data supported the synergistic effect of IL-1 $\beta$ and hyperglycemia in autophagy. However, it was noted that our data only discussed the specific situation of inflammation, which focused on the increase of IL-1 $\beta$. The diversity of inflammatory cytokines in an inflammatory microenvironment means our discussions are limited on a scope. More research should be designed to further discuss these problems.

\section{Conclusion}


This study confirmed that ADMSCs may have potential for use in improving cardiac function by restraining autophagy and apoptosis of myocardial cells. We also identified the roles of the IL-1 $\beta$ in hyperglycemia- and hyperlipidemia-induced cardiac injuries, which may be a key factor for diabetic complications.

\section{Abbreviations}

ADMSCs adipose-derived mesenchymal stem cells

BMSCs bone marrow-derived mesenchymal stem cells

CVD cardiovascular diseases

CXCL-15 interleukin-8

DM diabetes mellitus

EF ejection fraction

FS fractional shortening

HG high glucose

HF heart failure

HFD high-fat diet-fed

HFrEF educed left ventricular ejection fraction

IL-1 $\beta$ interleukin-1 $\beta$

IL-6 interleukin-6

LVEF lower left ventricular ejection fraction

LVEDD left ventricle (LV) at the end of diastole (LVEDD)

LVESD left ventricle (LV) at the end of systole (LVESD)

M macrophages

MI myocardial infarction

MSCs mesenchymal stem cells

NC normal chow 
STZ streptozotocin

T1DM type I diabetes mellitus

T2DM type II diabetes mellitus

TAM tumor-associated macrophages

TNFa tumor necrosis factor a

\section{Declarations}

\section{Ethics Statement}

All animal experimental procedures were carried out in accordance with the guidelines of the Shanghai Experimental Animal Center and the Shanghai Tenth People's Hospital (SYXK: 2014-0026) "People and Animal Use in Research" and in accordance with the principles set out in the Helsinki Declaration.

Experimental animals are treated humanely, and all efforts are made to alleviate their discomfort. All mice were housed in a specific pathogen-free animal vivarium and maintained on a $12 \mathrm{~h}$ light, $12 \mathrm{~h}$ dark cycle, with free access to standard rodent chow and water.

\section{Consent for publication}

Not applicable.

\section{Availability of Data and Materials}

All data generated or analysed during this study are included in this published article. The datasets used and/or analysed during the current study are available from the corresponding author on reasonable request.

\section{Competing interests}

The authors declare that there is no conflict of interests regarding the publication of this article.

\section{Funding}

This study is supported by grants No. 91939104 from the Chinese National Natural Science Foundation.

\section{Authors' contributions}

Xu xu and Lingyun Tao performed the animal experiments, cell function, and conducted western blot, immunofluorescence, and analyzed data. Lingyun Tao and JieZhou helped interpret the data and wrote the manuscript. Jing Ni conceived the project, designed experiments, analyzed data, interpreted results and wrote the manuscript. All authors read and approved the final manuscript. 
All contributors who do not meet the criteria for authorship should be list in an acknowledgments section.

\section{Acknowledgments}

Not applicable.

\section{References}

1. Kenny HC, Abel ED. Heart Failure in Type 2 Diabetes Mellitus. Circulation research. 2019;124(1):12141.

2. Athithan L, Gulsin GS, McCann GP, Levelt E. Diabetic cardiomyopathy: Pathophysiology, theories and evidence to date. World journal of diabetes. 2019;10(10):490-510.

3. Mátyás C, Kovács A, Németh BT, Oláh A, Braun S, Tokodi M, Barta BA, Benke K, Ruppert M, Lakatos BK, Merkely B, Radovits T. Comparison of speckle-tracking echocardiography with invasive hemodynamics for the detection of characteristic cardiac dysfunction in type- 1 and type-2 diabetic rat models. Cardiovascular diabetology. 2018;17(1):13.

4. Chareonthaitawee P, Sorajja P, Rajagopalan N, Miller TD, Hodge DO, Frye RL, Gibbons RJ. Prevalence and prognosis of left ventricular systolic dysfunction in asymptomatic diabetic patients without known coronary artery disease referred for stress single-photon emission computed tomography and assessment of left ventricular function. American heart journal. 2007;154(3):567-74.

5. Singh RM, Waqar T, Howarth FC, Adeghate E, Bidasee K, Singh J. Hyperglycemia-induced cardiac contractile dysfunction in the diabetic heart. Heart Fail Rev. 2018;23(1):37-54.

6. O'Brien T, Nguyen TT, Zimmerman BR, Hyperlipidemia and diabetes mellitus, Mayo Clinic proceedings 73(10) (1998) 969-76.

7. Yamagishi S. Cardiovascular disease in recent onset diabetes mellitus. Journal of cardiology. 2011;57(3):257-62.

8. Sun M, Sun L, Huang C, Chen BC, Zhou Z. Induction of Macrophage M2b/c Polarization by Adipose Tissue-Derived Mesenchymal Stem Cells. Journal of immunology research. 2019;2019:7059680.

9. Zhang S, Picard MH, Vasile E, Zhu Y, Raffai RL, Weisgraber KH, Krieger M. Diet-induced occlusive coronary atherosclerosis, myocardial infarction, cardiac dysfunction, and premature death in scavenger receptor class B type I-deficient, hypomorphic apolipoprotein ER61 mice. Circulation. 2005;111(25):3457-64.

10. Minguell JJ, Erices A. Mesenchymal stem cells and the treatment of cardiac disease, Experimental biology and medicine. (Maywood NJ). 2006;231(1):39-49.

11. White SJ, Chong JJH. Mesenchymal Stem Cells in Cardiac Repair: Effects on Myocytes, Vasculature, and Fibroblasts. Clinical therapeutics. 2020;42(10):1880-91.

12. Zou L, Ma X, Lin S, Wu B, Chen Y, Peng C. Bone marrow mesenchymal stem cell-derived exosomes protect against myocardial infarction by promoting autophagy. Experimental therapeutic medicine. 2019;18(4):2574-82. 
13. Liu L, Jin X, Hu CF, Li R, Zhou Z, Shen CX, Exosomes Derived from Mesenchymal Stem Cells Rescue Myocardial Ischaemia/Reperfusion Injury by Inducing Cardiomyocyte Autophagy Via AMPK and Akt Pathways, Cellular physiology and biochemistry: international journal of experimental cellular physiology, biochemistry, and pharmacology 43(1) (2017) 52-68.

14. Maharlooei MK, Bagheri M, Solhjou Z, Jahromi BM, Akrami M, Rohani L, Monabati A, Noorafshan A, Omrani GR. Adipose tissue derived mesenchymal stem cell (AD-MSC) promotes skin wound healing in diabetic rats. Diabetes Res Clin Pract. 2011;93(2):228-34.

15. Yin Q, Xu N, Xu D, Dong M, Shi X, Wang Y, Hao Z, Zhu S, Zhao D, Jin H, Liu W. Comparison of senescence-related changes between three- and two-dimensional cultured adipose-derived mesenchymal stem cells. Stem Cell Res Ther. 2020;11(1):226.

16. Hu C, Zhao L, Li L. Current understanding of adipose-derived mesenchymal stem cell-based therapies in liver diseases. Stem Cell Res Ther. 2019;10(1):199.

17. Xu S, Zhou Z, Li H, Liu Z, Pan X, Wang F, Huang Y, Li X, Xiao Y, Pan J, Wang C, Li D. BMSCs ameliorate septic coagulopathy by suppressing inflammation in cecal ligation and puncture-induced sepsis, Journal of cell science 131(3) (2018).

18. Liang H, Ding X, Yu Y, Zhang H, Wang L, Kan Q, Ma S, Guan F, Sun T. Adipose-derived mesenchymal stem cells ameliorate acute liver injury in rat model of CLP induced-sepsis via sTNFR1. Experimental cell research. 2019;383(1):111465.

19. Heo J, Choi Y, H.J.S.c.i. Kim, Adipose-Derived Mesenchymal Stem Cells Promote M2 Macrophage Phenotype through Exosomes, 2019 (2019) 7921760.

20. Sun M, Sun L, Huang C, Chen B, Z.J.J.o.i.r. Zhou, Induction of Macrophage M2b/c Polarization by Adipose Tissue-Derived Mesenchymal Stem Cells, 2019 (2019) 7059680.

21. Peet $C$, Ivetic A, Bromage DI, Shah AM. Cardiac monocytes and macrophages after myocardial infarction. Cardiovascular research. 2020;116(6):1101-12.

22. Shah SJ, Kitzman DW, Borlaug BA, van Heerebeek L, Zile MR, Kass DA, Paulus WJ. PhenotypeSpecific Treatment of Heart Failure With Preserved Ejection Fraction: A Multiorgan Roadmap. Circulation. 2016;134(1):73-90.

23. Sano S, Oshima K, Wang Y, MacLauchlan S, Katanasaka Y, Sano M, Zuriaga MA, Yoshiyama M, Goukassian D, Cooper MA, Fuster JJ, Walsh K. Tet2-Mediated Clonal Hematopoiesis Accelerates Heart Failure Through a Mechanism Involving the IL-1 $\beta /$ NLRP3 Inflammasome. J Am Coll Cardiol. 2018;71(8):875-86.

24. Everett BM, Cornel JH, Lainscak M, Anker SD, Abbate A, Thuren T, Libby P, Glynn RJ, Ridker PM. AntiInflammatory Therapy With Canakinumab for the Prevention of Hospitalization for Heart Failure. Circulation. 2019;139(10):1289-99.

25. Xiang E, Han B, Zhang Q, Rao W, Wang Z, Chang C, Zhang Y, Tu C, Li C, Wu D. Human umbilical cordderived mesenchymal stem cells prevent the progression of early diabetic nephropathy through inhibiting inflammation and fibrosis. Stem Cell Res Ther. 2020;11(1):336. 
26. Dai R, Liu J, Cai S, Zheng C, Zhou X. Delivery of adipose-derived mesenchymal stem cells attenuates airway responsiveness and inflammation in a mouse model of ovalbumin-induced asthma. American journal of translational research. 2017;9(5):2421-8.

27. Hamada D, Maynard R, Schott E, Drinkwater C, Ketz J, Kates S, Jonason J, Hilton M, Zuscik M, Mooney RJA. rheumatology, Suppressive Effects of Insulin on Tumor Necrosis Factor-Dependent Early Osteoarthritic Changes Associated With Obesity and Type 2. Diabetes Mellitus. 2016;68(6):1392-402.

28. Dei Cas A, Khan S, Butler J, Mentz R, Bonow R, Avogaro A, Tschoepe D, Doehner W, Greene S, Senni M, Gheorghiade M. G.J.J.H.f. Fonarow, Impact of diabetes on epidemiology, treatment, and outcomes of patients with heart failure, 3(2) (2015) 136-45.

29. Rios FJ, Touyz RM, Montezano AC. Isolation and Differentiation of Murine Macrophages, Methods in molecular biology. (Clifton NJ). 2017;1527:297-309.

30. Emile JF, Abla O, Fraitag S, Horne A, Haroche J, Donadieu J, Requena-Caballero L, Jordan MB, AbdelWahab O, Allen CE, Charlotte F, Diamond EL, Egeler RM, Fischer A, Herrera JG, Henter JI, Janku F, Merad M, Picarsic J, Rodriguez-Galindo C, Rollins BJ, Tazi A, Vassallo R, Weiss LM. Revised classification of histiocytoses and neoplasms of the macrophage-dendritic cell lineages. Blood. 2016;127(22):2672-81.

31. Hahn O, Ingwersen LC, Soliman A, Hamed M, Fuellen G, Wolfien M, Scheel J, Wolkenhauer O, Koczan D, Kamp G, Peters K. TGF- $\beta 1$ Induces Changes in the Energy Metabolism of White Adipose TissueDerived Human Adult Mesenchymal Stem/Stromal Cells In Vitro, Metabolites 10(2) (2020).

32. Shree N, Venkategowda S, Venkatranganna M, Datta I. R.J.I.j.o.o. Bhonde, Human adipose tissue mesenchymal stem cells as a novel treatment modality for correcting obesity induced metabolic dysregulation, 43(10) (2019) 2107-2118.

33. He C, Bassik MC, Moresi V, Sun K, Wei Y, Zou Z, An Z, Loh J, Fisher J, Sun Q, Korsmeyer S, Packer M, May HI, Hill JA, Virgin HW, Gilpin C, Xiao G, Bassel-Duby R, Scherer PE. B. Levine, Exercise-induced BCL2-regulated autophagy is required for muscle glucose homeostasis. Nature. 2012;481(7382):511-5.

34. Lee IH, Kawai Y, Fergusson MM, Rovira II, Bishop AJ, Motoyama N, Cao L, Finkel T, Atg7 modulates p53 activity to regulate cell cycle and survival during metabolic stress, Science (New York, N.Y.) 336(6078) (2012) 225-8.

35. Weindel CG, Richey LJ, Bolland S, Mehta AJ, Kearney JF, Huber BT. B cell autophagy mediates TLR7dependent autoimmunity and inflammation. Autophagy. 2015;11(7):1010-24.

36. Ju L, Han J, Zhang X, Deng Y, Yan H, Wang C, Li X, Chen S, Alimujiang M, Li X, Fang Q, Yang Y, Jia W. Obesity-associated inflammation triggers an autophagy-lysosomal response in adipocytes and causes degradation of perilipin 1. Cell death disease. 2019;10(2):121.

37. Muriach M, Flores-Bellver M, Romero FJ, Barcia JM. Diabetes and the brain: oxidative stress, inflammation, and autophagy, Oxidative medicine and cellular longevity 2014 (2014) 102158. 
38. Kolattukudy PE, Niu J. Inflammation, endoplasmic reticulum stress, autophagy, and the monocyte chemoattractant protein-1/CCR2 pathway. Circulation research. 2012;110(1):174-89.

\section{Tables}

Tabel 1 Primes Sequence

\begin{tabular}{|ll|}
\hline Gene & Sequence \\
\hline TNF-a (mouse) & Forward5'CAGGCGGTGCCTATGTCTC3' \\
& \\
\hline CXCL15 (mouse) & Forward5'TCGAGACCATTTACTGCAACAG3' \\
\hline IL-6 (mouse) & Reverse5'CATTGCCGGTGGAAATTCCTT3' \\
\hline & Reverse5'AGTGGTATAGACAGGTCTGTTGG3' \\
\hline
\end{tabular}

\section{Figures}


Figure 1

A

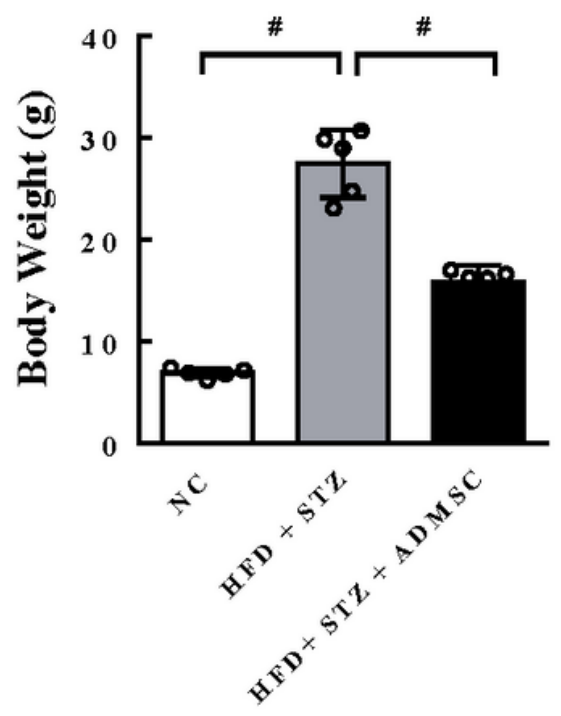

D

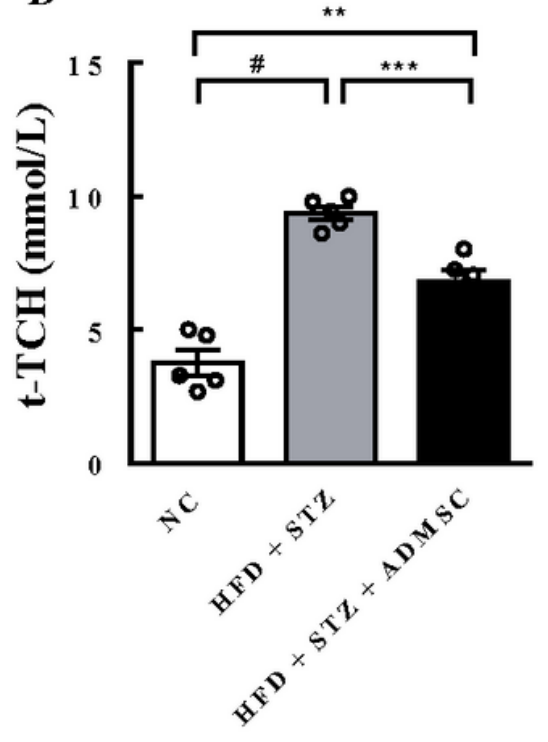

B

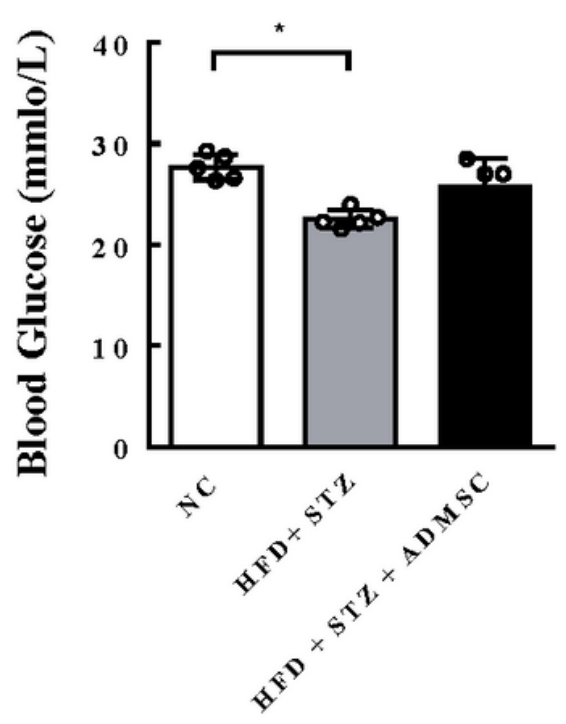

$\mathbf{E}$

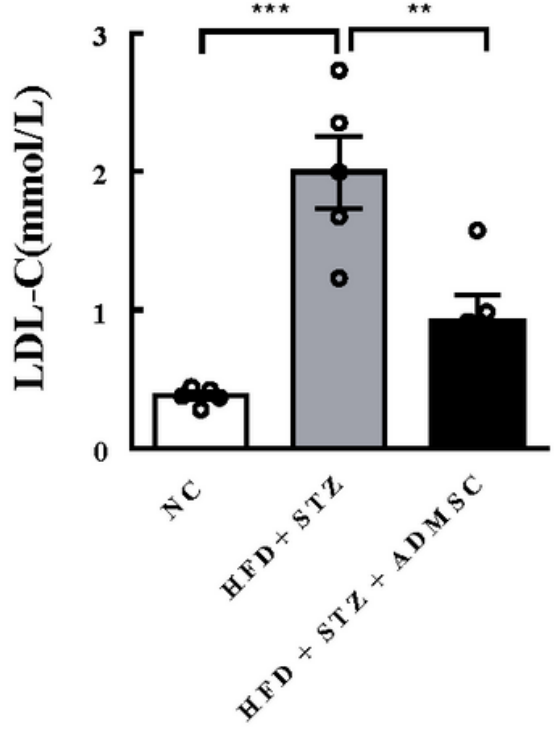

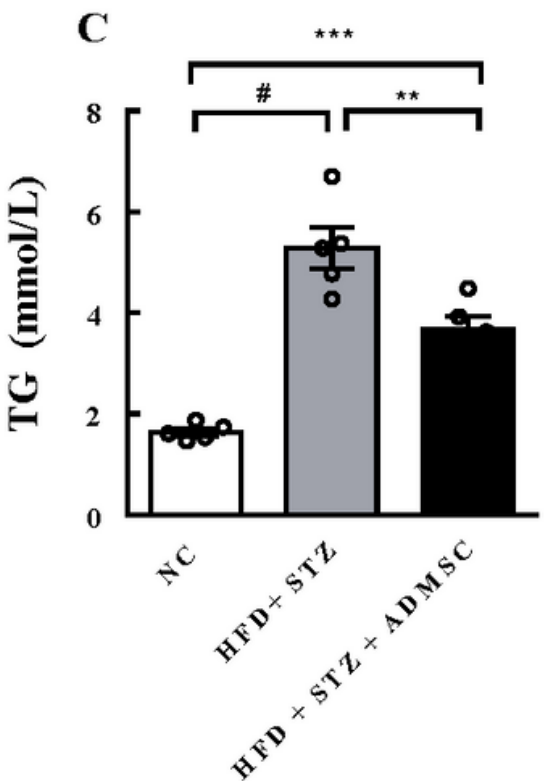

$\mathbf{F}$

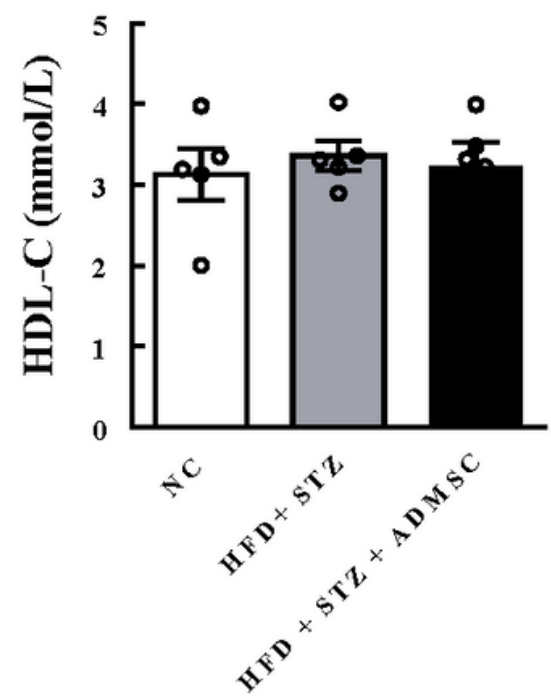

Figure 1

Adipose-derived mesenchymal stem cells (ADMSCs) improved glycometabolism and lipid metabolism in high-fat diet/STZ-induced diabetic mice. (A) Body weight ( $\mathrm{n}=6$ mice/group). (B) Blood glucose ( $\mathrm{n}=6$ mice/group). (C) Serum triglyceride ( $n=6$ mice/group). (D) Total cholesterol ( $n=6$ mice/group). (E) Lowdensity lipoprotein cholesterol ( $n=6$ mice/group) (F) High-density lipoprotein cholesterol $(n=6$ mice/group). NC, wild type mice with normal chow; HFD+STZ mice, high-fat diet/STZ-induced diabetic; HFD+STZ+ADMSCs mice, high-fat diet/STZ-induced diabetic mice injected with ADMSCs. Data are mean \pm SEM, One-way ANOVA with Bonferroni post-test, ${ }^{*} \mathrm{P}<0.05,{ }^{*} \mathrm{P}<0.01,{ }^{\star} * \mathrm{P}^{2}<0.005, \# \mathrm{P}<0.0001$. 
Figure 2
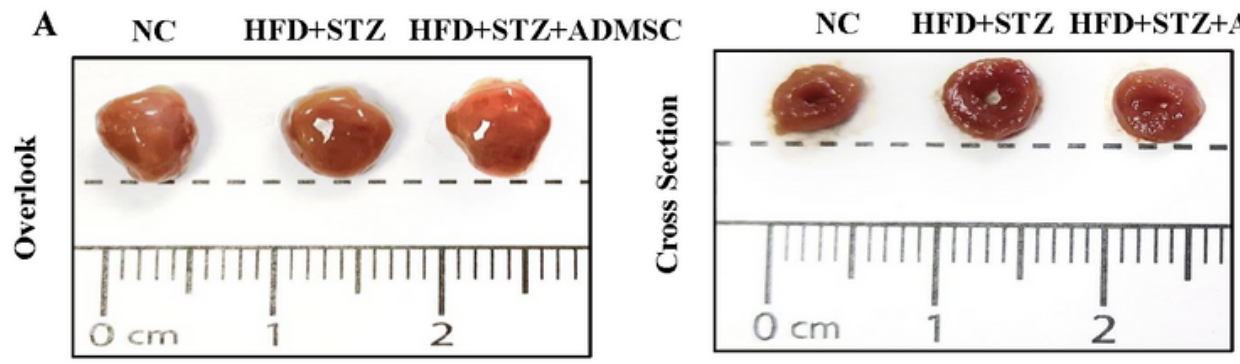

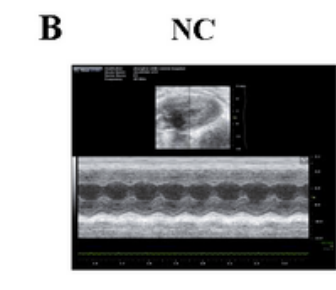

HFD+STZ

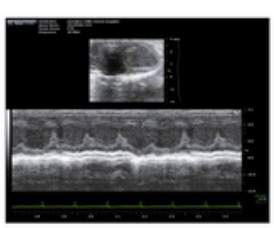

$\mathbf{E}$
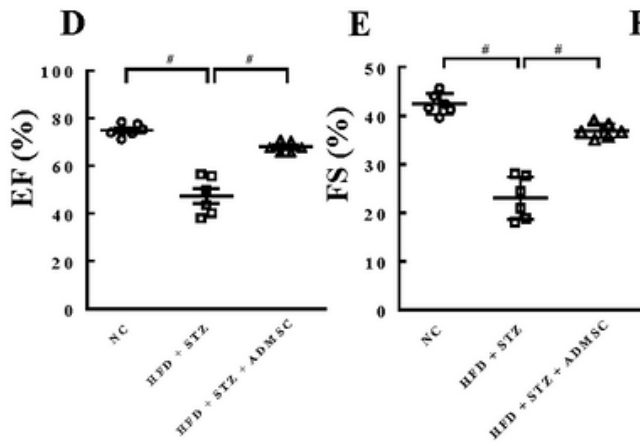

$\mathrm{H} \quad \mathrm{NC}$

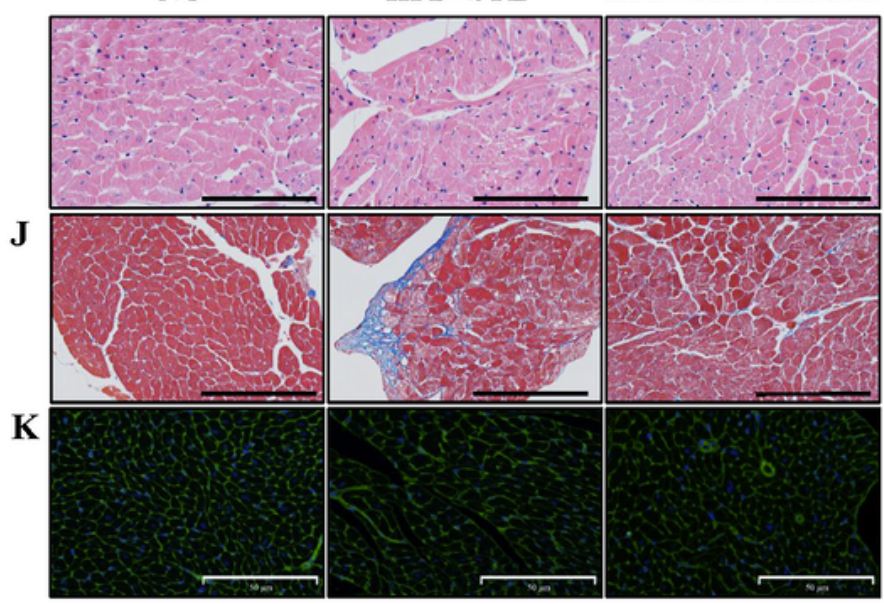

F
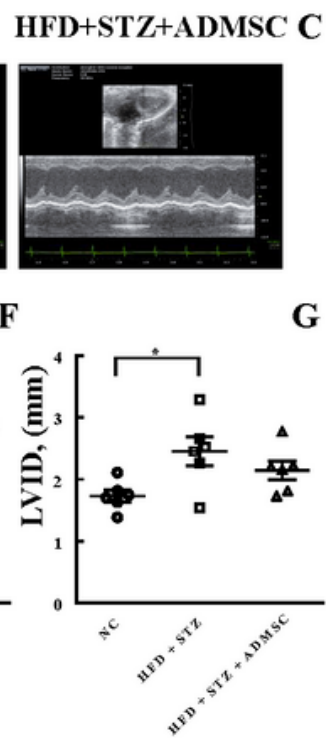

G
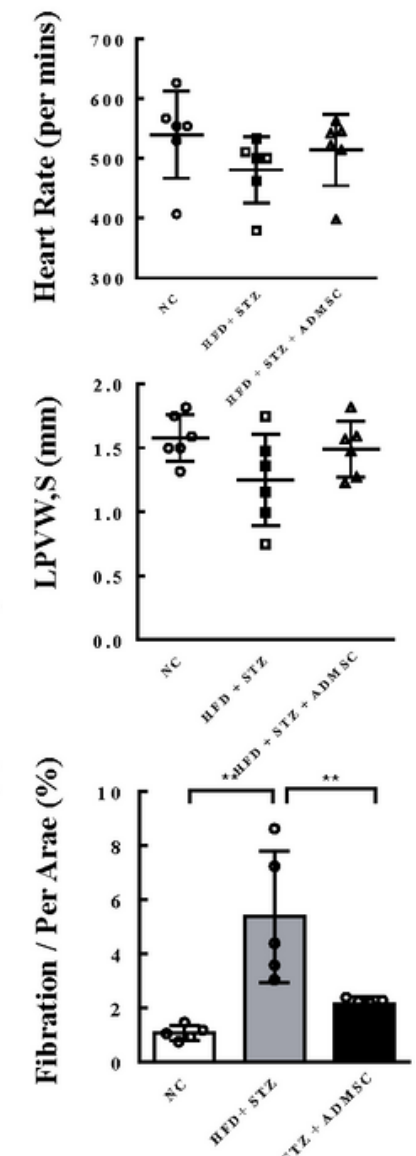

$\mathbf{L}$

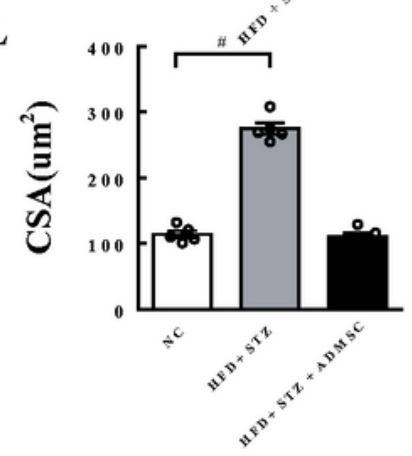

\section{Figure 2}

Adipose-derived mesenchymal stem cells (ADMSCs) alleviated heart failure in high-fat diet/STZ-induced diabetic mice. (A) The overlook of hearts. (B) Representative M-mode traces from left ventricular short axis view ( $n=6$ mice/group) (C) Heart rates ( $n=6$ mice/group) (D) Ejection fraction ( $n=6$ mice/group) (E) Left ventricular short-axis shortening rate ( $\mathrm{n}=6$ mice/group) (F) Left ventricular internal dimension ( $\mathrm{n}=6$ mice/group) (G) End diastolic of the posterior wall of the left ventricle ( $n=6$ mice/group) (H) HE staining 
of heart tissue ( $n=4$ scopes/group). Scar bar, $50 \mu \mathrm{m}$. (I) Masson staining of heart tissue ( $\mathrm{n}=5$ scopes/group). Scar bar, $50 \mu \mathrm{m}$ (J)WGA staining of myocardial cells ( $\mathrm{n}=5$ scopes/group). Scar bar, $50 \mu \mathrm{m}$ $(K)$ and $(L)$ Representative quantification of $(J)$ and $(L)$. WT, wild type mice with normal diet; HFD+STZ mice, high-fat diet/STZ-induced diabetic; HFD+STZ+ADSCs mice, high-fat diet/STZ-induced diabetic mice injected with ADSCs. Data are mean $\pm S E M$, One-way ANOVA with Bonferroni post-test, ${ }^{*} P<0.05$, $\star * P<0.01, * * * P<0.005, \# P<0.0001$.

\section{Figure 3}

A

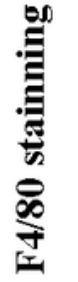

B

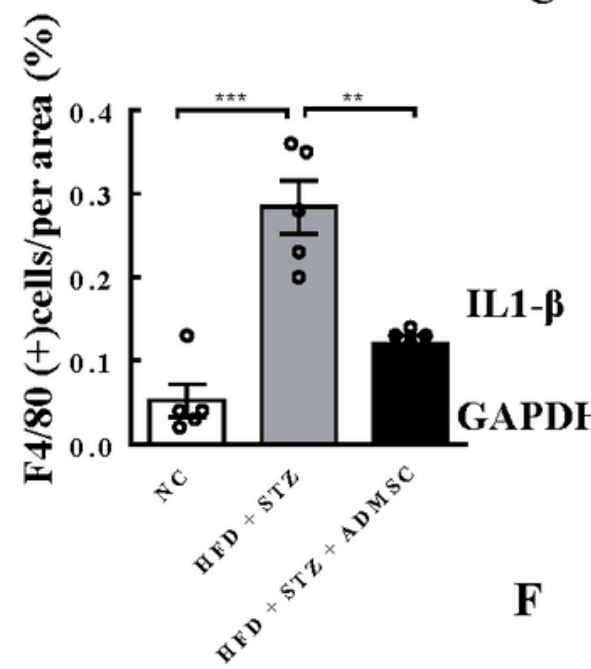

TNF

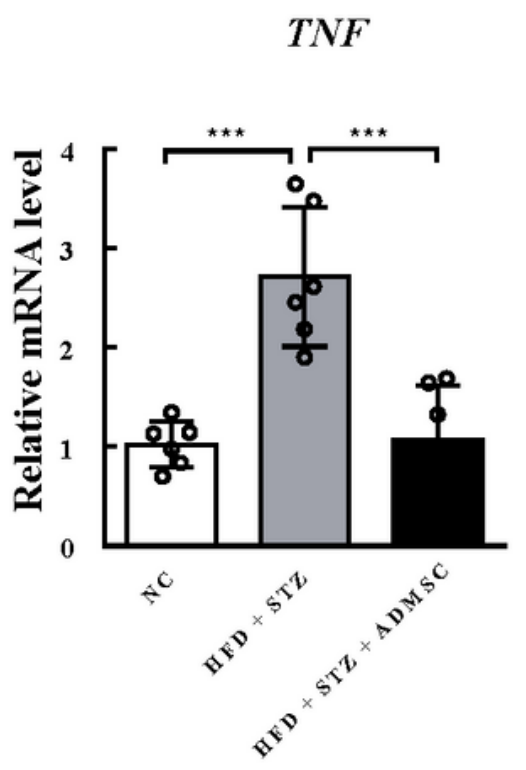

HFD+STZ

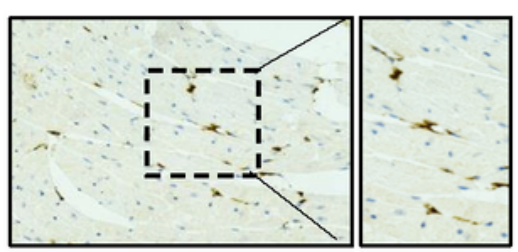

C
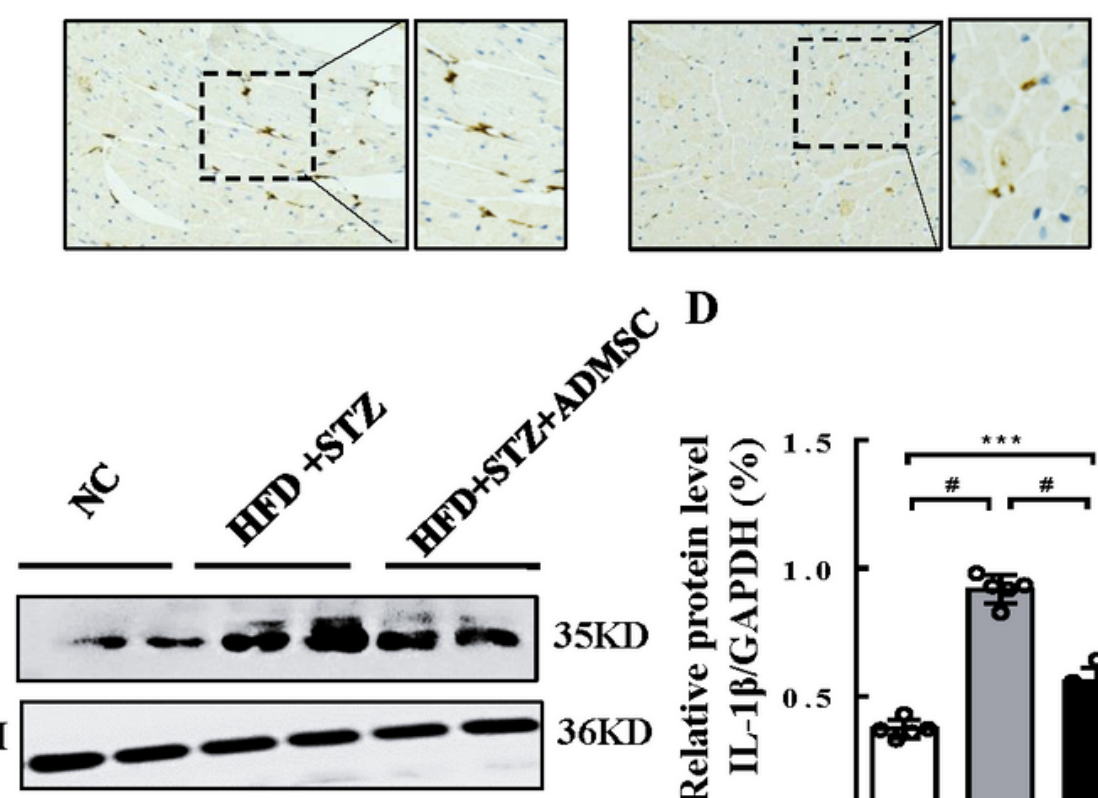

D

HFD +ST Z+ADMSC
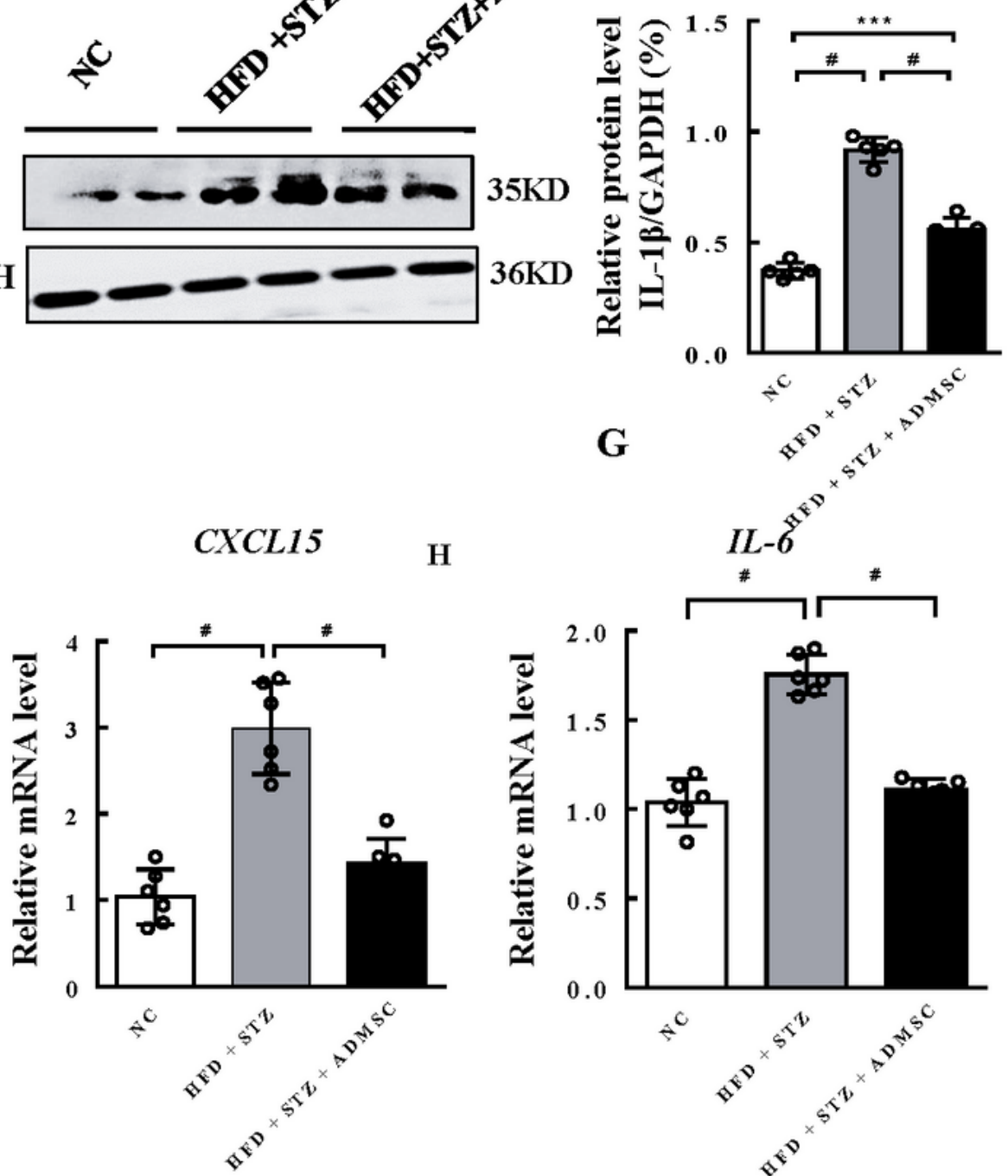

G

H

$35 \mathrm{KD}$

$36 \mathrm{KD}$

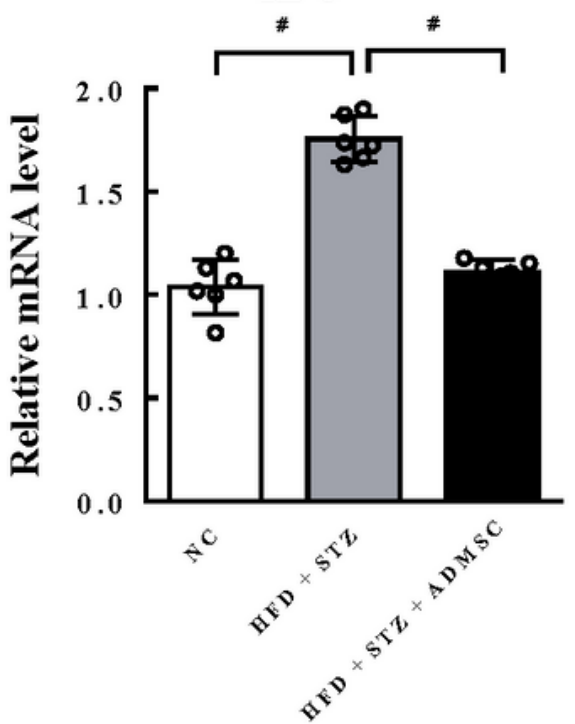

Figure 3 


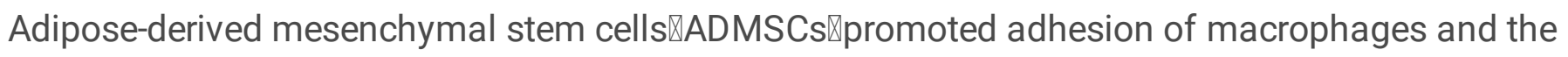
secretion of IL-1 $\beta$ in vivo and in vitro. (A) IHC staining of $F 4 / 80$ in cardiac tissue ( $n=5$ mice/group, 4 scopes/mice,). Scar bar, $100 \mu \mathrm{m}$. (B) Quantification of (A). (C) The expression of IL-1 $\beta$ in mice of three groups. The protein level was detected by western blot analysis ( $n=5 /$ group). (D) Quantification of (C). (E) The mRNA level of TNFa, CXCL15 and IL- 6 in cardiac tissue of three groups ( $n=5$ mice/group). (F) Schematic diagram for the co-culture system of ADMSCs and macrophages. NC, wild type mice with normal diet; HFD+STZ mice, high-fat diet/STZ-induced diabetic; HFD+STZ+ADMSCs mice, high-fat diet/STZ-induced diabetic mice injected with ADMSCs. TNFa, tumor necrosis factor a; IL-8, interleukin 8; IL-6, interleukin 6; HG, high glucose. Data are mean \pm SEM, One-way ANOVA with Bonferroni post-test, ${ }^{\star} \mathrm{P}<0.05,{ }^{\star *} \mathrm{P}<0.01,{ }^{\star *} \mathrm{P}<0.005, \# \mathrm{P}<0.0001$. 
Figure 49 c2 cells

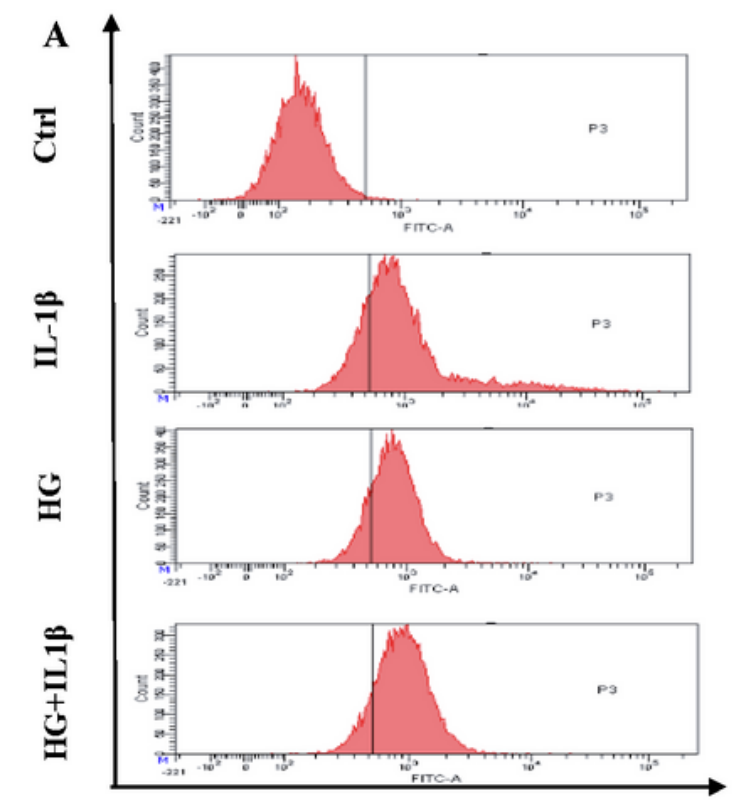

B

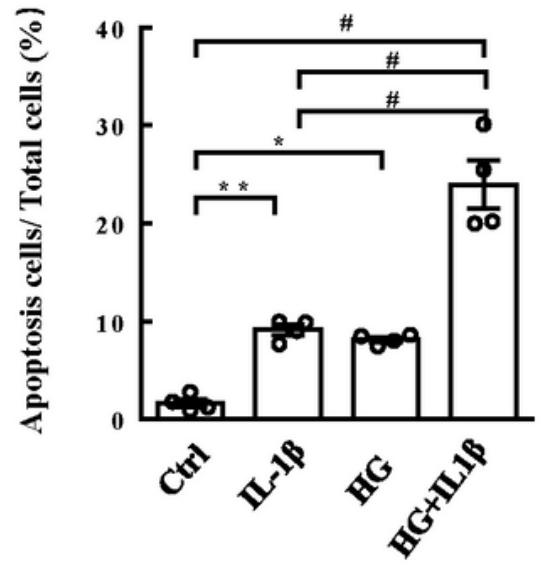

C

H9c2 cells

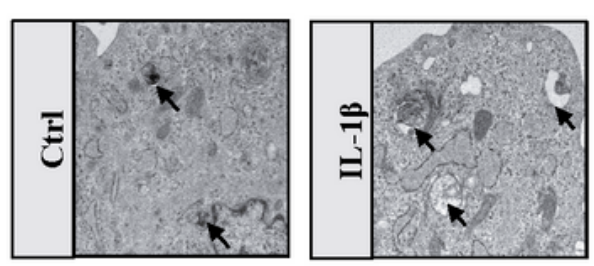

D c $\boldsymbol{y}^{\mathrm{s}}$
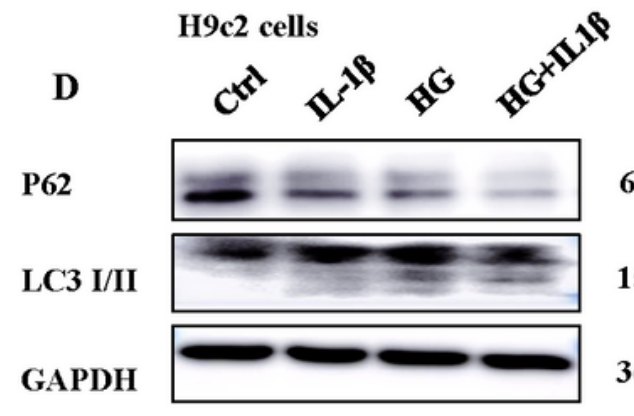

$62 \mathrm{kDa}$

15/10 kDa

$36 \mathrm{kDa}$
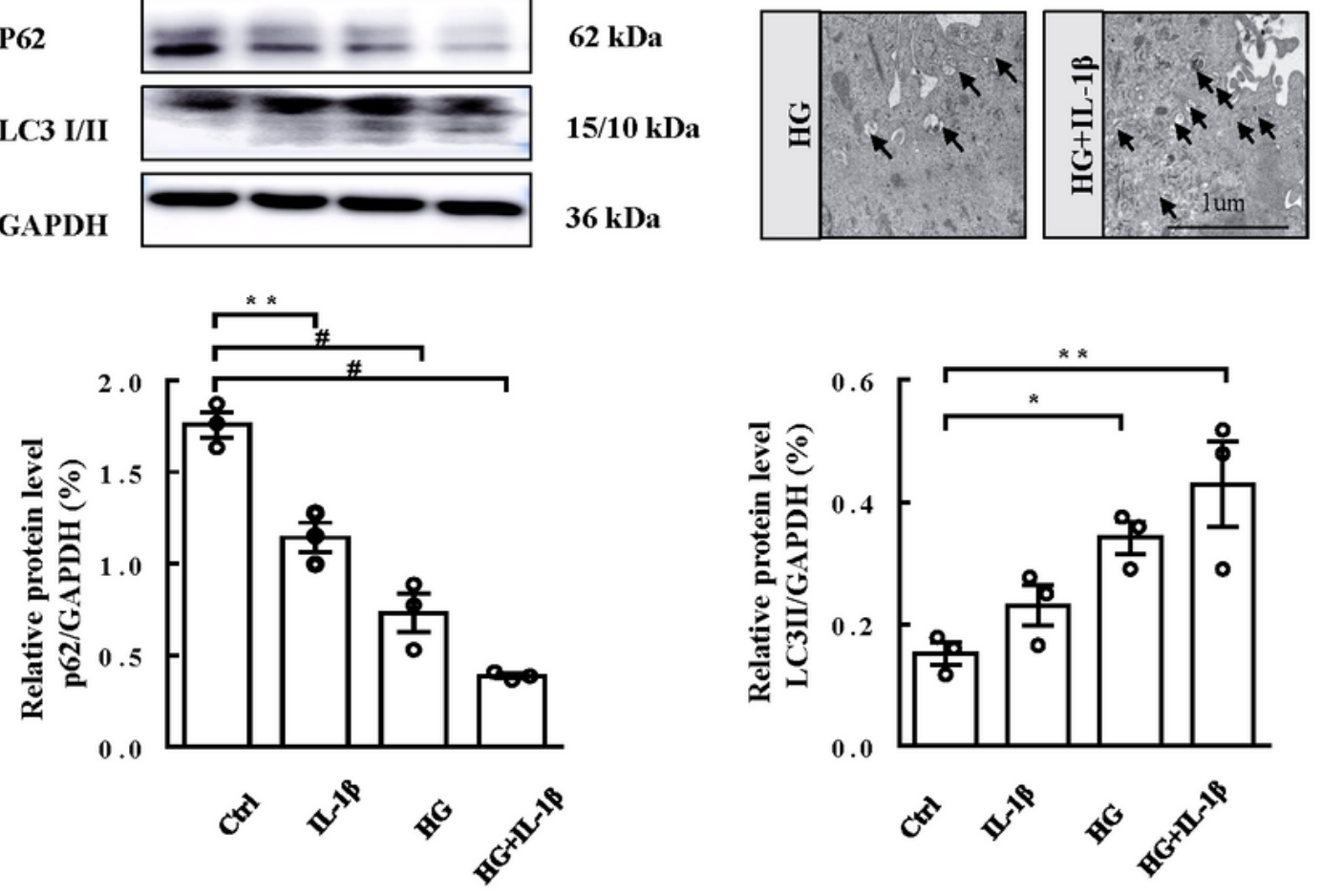

Figure 4

IL-1 $\beta$ enhanced diabetic cardiomyopathy by inhibiting autophagy and increasing apoptosis in H9c2 cells. (A) Apoptosis of H9c2 were measured by flow cytometry. (B) Quantification of (A). (C)The transmission electron microscope of autophagic vacuolization was decreased in IL-1 $\beta$ and HG stimulated H9c2 (magnification, $\times 5000, \times 15,000$ ). (D) The protein level of LC3I/II, P62 and Beclin1 in control and treated 
groups ( $n=4 /$ group). (E) Quantification of (D). Data are mean $\pm S E M$, One-way ANOVA with Bonferroni post-test, ${ }^{*} \mathrm{P}<0.05,{ }^{* *} \mathrm{P}<0.01,{ }^{*} * \mathrm{P}<0.005, \# \mathrm{P}<0.0001$.

Figure 5
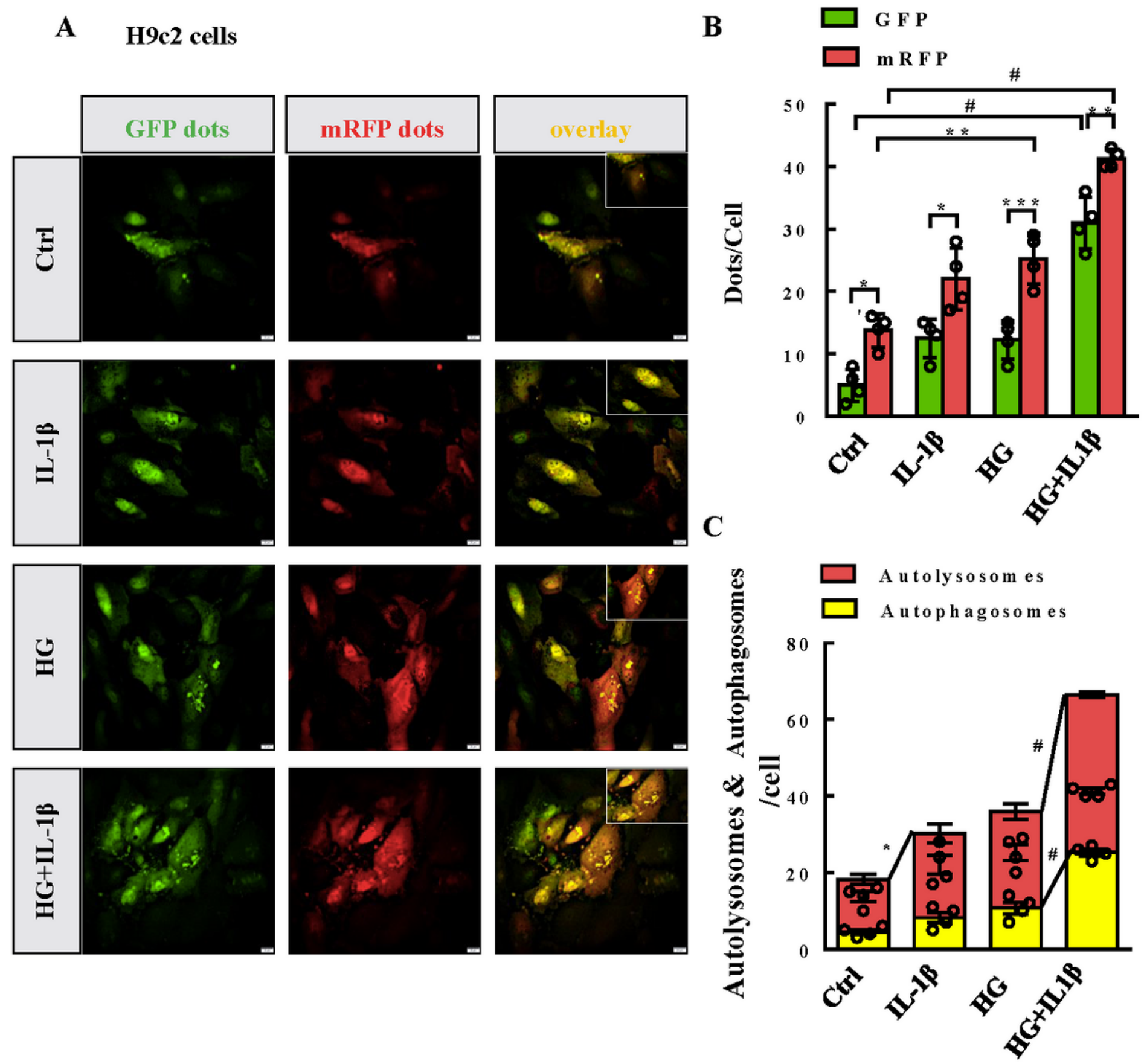

Figure 5

(A) Autophagosomes and autolysosomes were detected by tandem fluorescent mRFG-GFP-LC3 assay ( $n=4$ scopes/group). Yellow foci represent autophagosomes (GFP and mRFP overlayed), red foci represent autolysosome. Scar Bar, $10 \mu \mathrm{m}$. (B)\&(C) Quantification of (A). Data are mean $\pm S E M$, two-way ANOVA with Bonferroni post-test, $* P<0.05,{ }^{*} P<0.01, * \star \star P<0.005, \# P<0.0001$. 


\section{Supplementary Files}

This is a list of supplementary files associated with this preprint. Click to download.

- supplied.docx 